\title{
COMPARANDO MATRIZES DE MATEMÁTICA DO SAEB E DO NAEP
}

WALLACE NASCIMENTO PINTO JUNIOR

* Uma versão preliminar deste artigo está publicada nos anais do III Congresso Nacional de Avaliação em Educação, que ocorreu na Universidade Estadual Paulista "Júlio de Mesquita Filho", em Bauru, nos dias 22 a 24 de setembro de 2014

\section{RESUMO}

Este artigo tem como objetivo geral analisar as semelhanças $e$ diferenças estruturais das matrizes do Sistema de Avaliação da Educação Básica (Saeb) e do National Assessment of Educational Progress (Naep), e como específico comparar os descritores do tema "Espaço e Forma" testados no $9^{\circ}$ ano do Saeb com os objetivos da área Geometria testados na $8^{a}$ série do Naep, para produzir informações sobre as tendências curriculares que subjazem as escolhas das habilidades aferidas nesses sistemas de avaliação e que poderiam direcionar a atualização das matrizes de Matemática do Saeb. Os resultados sugerem que ambas as matrizes se assemelham na divisão dos objetos de conhecimento por áreas de conteúdo afins e na abordagem de variadas operações cognitivas; por outro lado, são distintas as formas pelas quais os descritores são apresentados em cada matriz. Notam-se algumas semelhanças entre as habilidades que são aferidas em ambos os testes na análise dos descritores do tema "Espaço e Forma".

PALAVRAS-CHAVE MATEMÁTICA • SISTEMA dE AVALIAÇÃO DA EDUCAÇÃO BÁSICA • NATIONAL ASSESSMENT OF EDUCATIONAL PROGRESS • CURRÍCULOS. 


\section{RESUMEN}

Este artículo tiene el propósito amplio de analizar las similitudes y diferencias estructurales de las matrices del Sistema de Evaluación de laEducación Básica (Saeb) y del National Assessment of Educational Progress (Naep), y específico de comparar los descriptores del tema "Espacio y Forma" testeados en el $9^{\circ}$ año del Saeb con los objetivos del área Geometría testeados en el $8^{\circ}$ año del Naep; ello para producir informaciones sobre las tendencias curriculares que subyacen en las elecciones de las habilidades verificadas en estos sistemas de evaluación y que podrían servir para actualizar las matrices de Matemáticas del Saeb. Los resultados sugieren que ambas matrices se asemejan en la división de los objetos de conocimiento por áreas de contenido afines y en el abordaje de varias operaciones cognitivas; por otro lado, son distintas las formas por las cuales los descriptores se presentan en cada matriz. Se notan algunas semejanzas entre las habilidades verificadas en ambas pruebas en el análisis de los descriptores del tema "Espacio y Forma".

PALABRAS CLAVE MATEMÁTICAS • SISTEMA DE EVALUACIÓN DE EDUCACIÓN BÁSICA - NATIONAL ASSESSMENT OF EDUCATIONAL PROGRESS • CURRÍCULOS.

\section{ABSTRACT}

This article aims to analyze the structural similarities and differences of the frameworks of the Evaluation System of Basic Education (Saeb) and the National Assessment of Educational Progress (Naep). More specifically, it seeks to compare the descriptors of the theme "Space and Shape", tested on the ninth grade of the Saeb, with those of Geometry, tested on the eighth grade of the Naep. This evaluation seeks to produce information on curricular trends which underlie the choices of skills measured in these assessment systems, that could guide the updating of the mathematical frameworks of the Saeb. The results suggest that both frameworks are similar in the separation of knowledge objects by related content areas and in addressing various cognitive operations; on the other hand, the ways in which the descriptors are presented in each matrix are different. Some similarities between the skills measured in both tests in the analysis of the descriptors of the theme "Space and Shape" can be observed. 
1 Sistema de Avaliação da Educação Básica, National Assessment of Educacional Progress (Avaliação Nacional do Progresso Educacional. tradução nossa), Programme for International Student Assessment Trends in International Mathematics and Science Study, Terceiro Estudo Regional Comparativo e Explicativo.

2 Deste ponto do artigo em diante, como abreviatura, vamos nos referir ao $5^{\circ}$ ano do ensino fundamental como $5 \mathrm{EF}$, ao $9^{\circ}$ ano como $9 \mathrm{EF}$ e à $3^{\circ}$ série do ensino médio como 3EM.

\section{INTRODUÇÃO}

Este artigo descreve parte de um estudo que está sendo realizado para comparar as matrizes de Matemática de algumas avaliações em larga escala (Saeb, Naep, Pisa, TIMSS e Terce), ${ }^{1}$ com vistas ao processo de atualização das matrizes do Saeb, as quais datam de 2001. A necessidade de revisar e atualizar essas matrizes se deve às mudanças de caráter epistemológico que vêm sendo incorporadas às diretrizes e aos documentos curriculares, e que afetam diretamente as habilidades aferidas pelo teste. Para o escopo deste artigo, apresentaremos comparativos apenas entre as matrizes do Saeb, o qual avalia os $5^{\circ}$ e $9^{\circ}$ anos do ensino fundamental e a $3^{\mathrm{a}}$ série do ensino médio, ${ }^{2}$ e as matrizes do Naep, o qual avalia as $4^{\mathrm{a}}, 8^{\mathrm{a}} \mathrm{e}$ $12^{\mathrm{a}}$ séries do sistema educacional dos Estados Unidos.

A escolha pelo comparativo das matrizes do Saeb com as do Naep se deu em razão das semelhanças estruturais entre elas e também porque este último sistema avaliativo tem um processo de implementação mais antigo do que o primeiro - enquanto o Saeb foi aplicado pela primeira vez em 1990 e suas matrizes ainda não estavam definidas, nesse mesmo 
ano, o Naep já havia estabelecido estruturas ${ }^{3}$ para o teste de Matemática, que só vieram a ser modificadas em 2005 e, mesmo assim, somente para a $12^{\mathrm{a}}$ série.

Nosso objetivo geral é analisar as semelhanças e diferenças estruturais das matrizes de Matemática desses dois testes, e nosso objetivo específico é comparar os descritores do tema Espaço e Forma medidos no 9EF do Saeb com os objetivos $^{4}$ da área Geometria medidos na $8^{\mathrm{a}}$ série do Naep. Almejamos com esse comparativo produzir algumas informações sobre as principais tendências curriculares que subjazem as escolhas das habilidades aferidas no Naep e que poderiam direcionar a atualização das matrizes de Matemática do Saeb.

\section{VISÃO GERAL DAS AVALIAÇÕES E SUAS MATRIZES}

\section{SAEB}

O Saeb ${ }^{5}$ está sob a responsabilidade do Instituto Nacional de Estudos e Pesquisas Educacionais Anísio Teixeira (Inep) e visa, segundo os documentos oficiais, a oferecer subsídios concretos para a formulação, a reformulação e o monitoramento das políticas públicas voltadas para a educação básica, além de oferecer dados e indicadores que possibilitem maior compreensão dos fatores que influenciam o desempenho dos alunos nas áreas e nos anos avaliados.

O Saeb teve sua primeira aplicação em 1990 e, desde 2013, há três avaliações que o compõem: a Avaliação Nacional da Educação Básica (Aneb), a Avaliação Nacional do Rendimento Escolar (Anresc, também denominada "Prova Brasil”) e a Avaliação Nacional da Alfabetização (ANA). A Aneb e a Anresc possuem as mesmas matrizes - exceto a da 3EM, pois não é uma etapa avaliada pela Anresc - e são realizadas bianualmente, enquanto a ANA possui uma matriz própria e é de realização anual.

Neste trabalho, a terminologia "matrizes de Matemática do Saeb" se refere especificamente às matrizes de Matemática da Aneb - e consequentemente às matrizes em comum com a Anresc. É importante ressaltar que a Aneb abrange, de maneira amostral, alunos das redes públicas e privadas do país, em áreas urbanas e rurais, matriculados no 5EF e 9EF e na 3EM,
3 Tradução nossa para frameworks.

4 Termo utilizado no Naep que possui mesmo significado que o termo descritor
5 Informações extraídas do site do Inep. Disponível em: <http://portal. inep.gov.br/web/saeb/aneb-e-anresc>. Acesso em: 01 ago. 2014. 
6 Tradução nossa para Nationa Center for Education Statistics.

7 Tradução nossa para Nationa! Assessment Governing Board. e apresenta os resultados do país como um todo, das regiões geográficas e das unidades da federação.

As matrizes do Saeb foram criadas em 1997 e, em 2001, houve a reorganização das matrizes de Língua Portuguesa e Matemática, as quais foram revistas por especialistas nas áreas de currículo, psicologia do conhecimento, língua portuguesa e matemática, e submetidas à validação por especialistas das diferentes secretarias de educação de todos os estados brasileiros e por amostra representativa de professores.

\section{NAEP}

O Naep é o instrumento utilizado pelos Estados Unidos para obter informações nacionalmente representativas e de forma contínua sobre o que os estudantes americanos sabem e podem fazer, e é conhecido como Boletim Escolar da Nação. O Centro Nacional de Estatísticas da Educação ${ }^{6}$ (NCES, em inglês) do Departamento de Educação dos EUA é o responsável pela realização do Naep, enquanto o Conselho Administrativo de Avaliação Nacional ${ }^{7}$ (NAGB, em inglês) define sua política e é responsável pelo desenvolvimento das estruturas (frameworks, em inglês) e das especificações do teste que servem de modelo para as avaliações. Essas estruturas fornecem a base teórica para os testes, descrevem o tipo de itens que devem ser incluídos e determinam o conteúdo a ser medido. Portanto, neste trabalho, a terminologia "matrizes de Matemática do Naep" se refere aos quadros que descrevem o conteúdo a ser aferido no teste de Matemática do Naep.

Ele foi realizado pela primeira vez em 1969 e, desde então, vem periodicamente colhendo e reportando dados sobre o desempenho em Leitura, Matemática, Ciências e outras disciplinas para alunos na $4^{\mathrm{a}}, 8^{\mathrm{a}}$ e $12^{\mathrm{a}}$ séries (grades, em inglês). O Naep tem como base amostras representativas de estudantes das séries supracitadas e fornece resultados sobre populações de estudantes (ex: todos que estão na $4^{\mathrm{a}}$ série) e grupos dentro dessas populações (ex: estudantes do sexo feminino, estudantes latinos) para o país como um todo, para os estados e para distritos urbanos selecionados.

De 1990 a 2003, vigoraram estruturas para o teste de Matemática e, embora elas sejam atualizadas periodicamente, 
os objetos de conhecimento de Matemática para as $4^{\mathrm{a}}$ e $8^{\mathrm{a}}$ séries não foram alterados, permitindo que o desempenho dos alunos pudesse ser comparado com anos anteriores. Em 2005, o Conselho Administrativo adotou novas estruturas para o teste de Matemática da $12^{\mathrm{a}}$ série a fim de refletir as mudanças nos padrões de ensino médio (high school, em inglês). Outras alterações foram feitas em 2009 para facilitar o relatório sobre a preparação desses estudantes para a educação e para a formação pós-secundária.

As estruturas estabelecidas pelo NAGB para todas as disciplinas do Naep, incluindo Matemática, baseiam-se na colaboração de uma vasta gama de especialistas e no envolvimento de representantes de empresas, formuladores de políticas públicas municipais e estaduais, especialistas em currículo de órgãos de educação estaduais e municipais, profissionais, pesquisadores e educadores.

\section{METODOLOGIA}

A metodologia que utilizamos neste estudo para comparar as matrizes de Matemática do Saeb e do Naep possui critérios muito semelhantes às metodologias utilizadas em Neidorf et al. (2006, p. 20) para comparação de itens entre Naep, TIMSS e Pisa, e em United States of America $(2013$, p. 7) para comparar as estruturas de Matemática da $8^{\mathrm{a}}$ série do Naep e do TIMSS. Embora, inicialmente, não os tenhamos tomado por base, encontramos nos trabalhos supracitados diretrizes para realizar este estudo comparativo.

Com proximidade da análise realizada entre a estrutura do Naep e do TIMSS (UNITED STATES OF AMERICA, 2013), as matrizes de Matemática do Naep e do Saeb também estão organizadas em torno de duas dimensões - uma dimensão de conteúdo (cujos elementos chamamos neste trabalho de "objetos de conhecimento") e uma dimensão cognitiva (cujos elementos chamamos de "operações cognitivas"). As interseções entre essas duas dimensões definem as habilidades a serem aferidas nos testes, as quais são especificadas por meio dos descritores no Saeb e objetivos no Naep. Tendo em vista que tanto o termo descritor, quanto o termo objetivo definem uma 
sentença que indica uma habilidade a ser aferida por um item do teste, então, consideramos essas sentenças como uma unidade comum entre as duas matrizes e, consequentemente, passíveis de serem comparadas.

Os descritores do Saeb foram comparados aos objetivos do Naep de acordo com os seguintes critérios:

1. (Nível macro) Etapas de escolaridade: Os descritores do 5EF, do 9EF e da 3EM do Saeb foram comparados aos objetivos das $4^{\mathrm{a}}, 8^{\mathrm{a}}$ e $12^{\mathrm{a}}$ séries do Naep, respectivamente.

2. (Nível intermediário) Temas e subtemas: Buscando-se a melhor correspondência entre descritores e objetivos, eles foram classificados de acordo com cinco temas (comuns entre as matrizes, embora no Saeb "Álgebra e Funções e Números e Operações" consistem em um único tema), e de acordo com os subtemas existentes nas matrizes Naep. Optamos por seguir essa subdivisão presente nas matrizes do Naep em razão da facilidade que ela proporcionou ao processo de classificação.

3. (Nível micro) Objeto do conhecimento e operação cognitiva: Cada descritor do Saeb foi relacionado a um objetivo do Naep, verificando-se as similaridades e diferenças entre o objeto de conhecimento e a operação cognitiva avaliada. Alguns descritores do Saeb foram relacionados a mais de um objetivo do Naep e vice-versa.

Além desses critérios, também foram tomadas as seguintes decisões:

- Considerar todos os temas da matriz, visto que um descritor de uma matriz poderia ser relacionado a um tema diferente na outra (ex: "Calcular a probabilidade de um evento" presente na matriz do Saeb foi classificado dentro do tema "Tratamento da Informação”, apesar de estar localizado no tema "Números e Operações/Álgebra e Funções").

- A classificação quanto ao objeto de conhecimento e à operação cognitiva deve prevalecer sobre a classificação 
quanto à etapa de escolaridade. Quando houve conflito, foi indicado que o descritor ou objetivo poderia ser localizado em outra etapa escolar.

- Em casos de operações cognitivas muito diferentes, relacionar operações que poderiam "estar contidas" em outras (ex: consideramos que "Identificar uma equação ou uma inequação de primeiro grau que expressa um problema" da matriz do Saeb poderia estar contida em "Escrever expressões algébricas, equações ou inequações para representar uma situação" da matriz do Naep, porque, para que um estudante seja capaz de escrever uma expressão algébrica, entendemos que é necessário que ele seja, antes, capaz de identificá-la).

Durante o processo de comparação, foram registradas observações acerca de características de descritores específicos e motivos que fundamentaram algumas classificações.

\section{COMPARAÇÕES ENTRE AS MATRIZES DE MATEMÁTICA DO SAEB E DO NAEP}

Nesta seção, apresentamos alguns resultados das comparações que foram feitas entre as matrizes de Matemática do Saeb e as do Naep, envolvendo a dimensão de conteúdo, a dimensão cognitiva e, a partir da confluência dessas dimensões, os descritores, que são as unidades comuns entre as duas matrizes. Em razão do número de páginas, neste artigo limitamos os resultados das comparações dos descritores ao tema "Espaço e Forma".

\section{DIMENSÃO DE CONTEÚDO}

Para as três etapas escolares avaliadas, a matriz do Saeb divide os objetos de conhecimento em quatro temas: "Espaço e Forma”; “Grandezas e Medidas”; “Números e Operações/Álgebra e Funções”; e "Tratamento da Informação", repetindo a mesma classificação dos Parâmetros Curriculares Nacionais. Há um total de 28 descritores no 5EF, 37 no 9EF e 35 na 3EM. Os descritores são listados para cada etapa escolar e, como 
não há nenhum quadro comparativo desses descritores, não ficam explícitas a continuidade e a complexidade das habilidades de uma etapa para a outra.

$\mathrm{Na}$ matriz do Naep, os objetos de conhecimento estão divididos em cinco áreas de conteúdo: "Propriedades Numéricas e Operações”; "Medidas"; "Geometria”; "Análise de Dados”, "Estatística e Probabilidade”; e "Álgebra”. Cada área de conteúdo é ainda dividida em subáreas, que incluem um conjunto de descritores específicos para cada série. Há um total de 65 descritores na $4^{\mathrm{a}}$ série, 100 na $8^{\mathrm{a}}$ série e 130 na $12^{\mathrm{a}}$ série. A organização das matrizes consiste em um único quadro, por meio do qual é possível identificar a continuidade e a complexidade das habilidades de uma série para a outra.

\section{DIMENSÃO COGNITIVA}

Matrizes que são construídas com referência na Taxonomia de Bloom revisada (ANDERSON et al., 2001) geralmente apresentam a estrutura de uma tabela, cujas células são as habilidades, criadas pela interseção de um objeto do conhecimento e uma das operações cognitivas: lembrar, compreender, aplicar, analisar, avaliar ou criar. Entretanto, nem a matriz do Saeb, nem a do Naep apresentam explicitamente tal estrutura, embora guardem algum paralelo com a Taxonomia de Bloom.

Ainda que várias operações cognitivas estejam presentes nos descritores da matriz do Saeb (ex: identificar, relacionar, resolver problema, etc.), eles não são organizados ou classificados de acordo com essas operações, mas apenas listados.

A partir de detalhes na redação de alguns descritores, é possível inferir o nível de complexidade em cada etapa escolar para uma determinada habilidade (ex: "Resolver problema envolvendo o cálculo do perímetro de figuras planas, desenhadas em malhas quadriculadas" no 5EF e "Resolver problema envolvendo o cálculo do perímetro de figuras planas" no 9EF). Porém, há vários descritores que apresentam a mesma redação, o que sugere que determinadas habilidades são aferidas com iguais níveis de complexidade, mesmo em etapas escolares distintas (ex: "Resolver problema envolvendo o cálculo de perímetro de figuras planas", "Resolver problema que envolva variações proporcionais, diretas ou inversas entre 
grandezas" e "Resolver problema que envolva equação de segundo grau" possuem a mesma redação tanto no 9EF quanto no 3EM).

A matriz do Naep define a dimensão cognitiva utilizando três níveis de complexidade: baixa, moderada e alta. Esses níveis de complexidade formam uma descrição ordenada das demandas que um item coloca sobre o raciocínio dos estudantes. Esses níveis hierárquicos são usados em combinação com os objetos de conhecimento para assegurar que o conjunto de itens que compõem a avaliação seja equilibrado, medindo desde a recordação de fatos e procedimentos matemáticos (baixa complexidade), uso e explicação de compreensão matemática conceitual e procedimental (complexidade moderada), até raciocínio com e sobre o conteúdo de matemática (alta complexidade) (UNITED STATES OF AMERICA, 2013).

Em cada um das áreas de conteúdo, na matriz do Naep, há uma subárea que se inicia com "Raciocínio matemático" (ex: Raciocínio matemático em geometria, Raciocínio matemático usando números). De forma geral, tomando a Taxonomia de Bloom revisada como referência (ANDERSON et al., 2001), essas subáreas envolvem as operações cognitivas "analisar" ou "avaliar", as quais não são contempladas nas matrizes de Matemática do Saeb.

É preciso levar em consideração que os itens do Naep podem ser (i) de múltipla escolha; (ii) de resposta curta (short constructed response); ou (iii) de resposta estendida (extended constructed response). Essa diversidade de tipos de itens implica ter mais opções de operações cognitivas a serem mobilizadas pelos participantes do teste e, em decorrência, mais habilidades podem ser medidas. Por outro lado, como no Saeb são utilizados apenas itens de múltipla escolha, então não é possível medir nesse teste habilidades envolvendo operações cognitivas como desenhar, esboçar, escrever, completar, montar, construir, propor, etc.

\section{ANÁLISES SOBRE O TEMA ESPAÇO E FORMA}

O tema "Espaço e Forma", classificação utilizada no Saeb, corresponde à área de conteúdo Geometria na matriz do Naep, a qual é organizada em cinco subáreas: 
- Dimensão e Forma;

- Transformação de formas e preservação de propriedades;

- Relações entre figuras geométricas;

- Posição, direção e geometria de coordenadas;

- Raciocínio matemático em geometria.

Conforme foi relatado na metodologia, optamos por seguir essa subdivisão presente nas matrizes do Naep porque trouxe facilidade ao processo de classificação.

No Quadro 1, em anexo, apresentamos uma síntese sobre o comparativo de descritores do $9^{\circ}$ ano do Saeb e da $8^{\mathrm{a}}$ série do Naep. Nele, a referência D01, por exemplo, indica que esse é o primeiro descritor na matriz do $9^{\circ}$ ano do Saeb, sendo sequencial. Por outro lado, 8.G.1.a, por exemplo, indica que o objetivo encontra-se na $8^{a}$ série do Naep, na área Geometria, na $1^{\mathrm{a}}$ subárea, sendo o objetivo "a” nessa subárea. Embora essa referência para os objetivos do Naep seja sequencial, não existem os objetivos 8.G.2.b, 8.G.3.a e 8.G.3.e na $8^{\mathrm{a}}$ série, porque as habilidades que seriam medidas por eles foram restritas à série anterior ou à série seguinte.

Ainda sobre o Quadro 1, as operações cognitivas foram destacadas em negrito e sublinhado, enquanto os objetos de conhecimento foram destacados em itálico. Os termos que estão apenas em negrito foram compreendidos como operações cognitivas paralelas à operação principal na redação do descritor. Em sua maioria, essas operações paralelas acompanham a operação "Resolver problema", indicando o modo como um determinado problema deve ser resolvido. As células vazias sombreadas na cor cinza claro indicam que não foi encontrada correspondência para o descritor entre as matrizes. Já as células sombreadas na cor cinza escuro significam que foi observada a localização de descritores em etapas escolares distintas.

Em termos numéricos, observa-se um total de 11 descritores no $9^{\circ}$ ano do Saeb e 21 objetivos na $8^{\mathrm{a}}$ série do Naep, o que sugere que o Naep mede uma gama maior de habilidades do que o Saeb. 
Observa-se em ambos os testes a presença dos seguintes objetos do conhecimento: figuras bidimensionais e tridimensionais, ângulos, círculos, ampliação/redução, propriedades dos polígonos (e, particularmente, de triângulos e quadriláteros), Teorema de Pitágoras e coordenadas cartesianas. Por um lado, não estão explícitos no Saeb: caminhos, simetrias, reflexões, rotações, translações, congruência, semelhança, posições relativas de pontos e retas no plano, seção transversal de um sólido. Por outro lado, no Naep não encontramos elementos de um círculo (raio, diâmetro, etc., os quais estão implícitos no descritor do Saeb: "Reconhecer círculo/circunferência, seus elementos e algumas de suas relações") e nem posição de objetos em mapas, croquis e outras representações gráficas.

Relativamente às operações cognitivas, apoiando-se apenas na redação dos descritores, em ordem de predominância, observa-se no Saeb as operações: identificar, reconhecer, resolver problema, relacionar e interpretar, ao passo que no Naep ocorrem: descrever, identificar, resolver problema, representar, reconhecer, desenhar, analisar, definir, classificar, demonstrar compreensão, fazer, testar, prever os resultados, justificar, aplicar, usar, visualizar. Portanto, as operações identificar, reconhecer e resolver problema estão presentes em ambos os recortes dessas matrizes, havendo maior variabilidade no Naep do que no Saeb. Esse último fato corrobora o que tratamos anteriormente sobre a relação entre tipos de itens e diversidade de operações cognitivas.

Os subtemas com maior correspondência entre os descritores das matrizes são "Dimensão e Forma" e "Relações entre figuras geométricas". Nenhum descritor do Saeb foi localizado no subtema "Raciocínio Matemático em Geometria", no qual são introduzidos na matriz do Naep elementos sobre demonstrações matemáticas, nesse caso, "Fazer e testar uma conjectura geométrica sobre polígonos regulares".

No subtema "Dimensão e Forma", estão presentes nas duas matrizes "figuras bidimensionais e tridimensionais", mas a maneira como esses objetos do conhecimento são abordados é diferente em cada teste. No Saeb, há um contexto restrito, no qual espera-se que o participante do teste 
seja capaz de "identificar propriedades comuns e diferenças" entre essas figuras e utilize essa habilidade para relacionar uma figura tridimensional com sua planificação. Já no Naep, é esperado que, além de identificar, outras operações cognitivas sobre essas figuras sejam mobilizadas em diversos contextos, tais como, "Identificar um objeto geométrico dada uma descrição escrita de suas propriedades", "Identificar, definir, ou descrever formas geométricas no plano e no espaço tridimensional dada uma representação visual”, "Representar ou descrever uma situação tridimensional em um desenho bidimensional a partir de diferentes vistas" e, ainda, "Demonstrar uma compreensão sobre as formas bi e tridimensionais em nosso mundo por meio de identificação, desenho, modelagem, construção ou decomposição".

Ainda no subtema "Dimensão e Forma", foi observada uma divergência entre etapas escolares nas habilidades que se referem a "ângulos" - enquanto o Saeb testa a habilidade "Reconhecer ângulos como mudança de direção ou giros, identificando ângulos retos e não retos" no 9EF, no Naep, uma habilidade similar "Identificar ou desenhar ângulos e outras figuras geométricas no plano" é aferida já na $4^{\mathrm{a}}$ série.

No subtema "Transformação de formas e preservação de propriedades", os descritores do Saeb que envolvem ampliação e/ou redução e transformação homotética talvez pudessem ser concentrados em um único descritor, por apresentarem habilidades com a mesma essência - que é reconhecer a conservação ou modificação de propriedades e/ou medidas em ampliação, redução ou em transformação homotética. Outras transformações geométricas - simetrias, reflexões, translações e rotações - não estão explícitas na matriz do Saeb. No Naep, essas transformações já estão presentes nas matrizes da $4^{\text {a }}$ série e, além de serem aprofundadas na $8^{\mathrm{a}}$ série, são introduzidas nesse subtema "Habilidades envolvendo as relações de congruência e semelhança”.

No subtema "Relações entre figuras geométricas", apesar de diferirem em algumas especificidades, ambas as matrizes incluem "Resolver problema utilizando propriedades dos polígonos" (o Saeb especifica soma de seus ângulos internos, número de diagonais, cálculo da medida de cada 
ângulo interno nos polígonos regulares), "Resolver problema utilizando o Teorema de Pitágoras" (o Saeb abrange o uso de outras relações métricas no triângulo retângulo), "Identificar propriedades de triângulos e quadriláteros" (o Naep requer descrever e analisar essas propriedades, ou seja, vai além da identificação). Somente o Naep inclui "Representar situações-problema com modelos geométricos para resolver problema" e "Descrever ou analisar propriedades e relações de retas paralelas ou concorrentes".

Finalmente, no subtema "Posição, direção e geometria de coordenadas", a matriz do Saeb conta com a habilidade "Identificar a localização/movimentação de objeto em mapas, croquis e outras representações gráficas”, a qual não está explícita na matriz do Naep. O Saeb introduz na $8^{\text {a }}$ série a habilidade "Interpretar informações apresentadas por meio de coordenadas cartesianas", a qual é coberta com maiores especificidades na $4^{\text {a }}$ série do Naep, embora esteja localizada na área de conteúdo álgebra. Além do que já foi analisado, nesse subtema somente a matriz do Naep contempla "Descrever posições relativas de pontos e retas [...]", "Descrever interseções de figuras geométricas no plano" e "Visualizar ou descrever a seção transversal de um sólido".

\section{CONSIDERAÇÕES FINAIS}

Este artigo teve como objetivo analisar as semelhanças e diferenças estruturais das matrizes de Matemática do Saeb e do Naep, além de comparar os descritores do tema "Espaço e Forma" testados no 9EF do Saeb com os objetivos da área Geometria testados na $8^{\mathrm{a}}$ série do Naep.

Estruturalmente, ambas as matrizes se assemelham na divisão dos objetos de conhecimento por temas ou áreas de conteúdo afins - exceto álgebra, que é uma área independente no Naep, mas no Saeb encontra-se junto ao tema "Números e Operações" - e na abordagem de variadas operações cognitivas. Por outro lado, são distintas as formas pelas quais os descritores são apresentados em cada matriz e, por causa dos tipos de itens que são utilizados, há mais opções de operações cognitivas no Naep do que no Saeb. 
Finalmente, ao comparar os descritores do tema "Espaço e Forma”, notam-se algumas semelhanças entre as habilidades que são aferidas em ambos os testes, principalmente nos subtemas "Dimensão e Forma" e "Relações entre figuras geométricas". Contudo, de forma geral, tanto a ausência no Saeb de objetos do conhecimento como simetrias, reflexões, rotações, translações, congruência, semelhança, quanto a abordagem de um conjunto restrito de operações cognitivas (identificar, reconhecer, resolver problema, relacionar e interpretar) sugerem um menor alinhamento às tendências curriculares em educação matemática do que o Naep.

\section{AGRADECIMENTOS}

Agradeço aos colegas João Luiz Horta Neto e Marco César Araújo Pereira pelas observações e sugestões que muito contribuíram para o desenvolvimento deste estudo.

\section{REFERÊNCIAS}

ANDERSON, L. W. et. al. A taxonomy for learning, teaching and assessing: a revision of Bloom's Taxonomy of Educational Objectives. New York: Addison Wesley Longman, 2001.

BRASIL. Instituto Nacional de Estudos e Pesquisas Educacionais. SAEB 2001: novas perspectivas. Brasília, DF: Inep, 2002.

NATIONAL ASSESSMENT GOVERNING BOARD. Mathematics Framework for the 2013 National Assessment of Educational Progress. Washington, DC: NAGB, 2012. Disponível em: <http://www.nagb.org/content/nagb/assets/documents/ publications/frameworks/math-2013-framework.pdf>. Acesso em: 28 jul. 2014.

NEIDORF, T. S.; BINKLEY, M.; GATTIS, K.; NOHARA, D. Comparing Mathematics Content in the National Assessment of Educational Progress (NAEP), Trends in International Mathematics and Science Study (TIMSS), and Program for International Student Assessment (PISA) 2003 Assessments (NCES 2006-029). Washington, DC: U.S. Department of Education. NCES, 2006. Disponível em: <http://nces.ed.gov/ pubsearch>. Acesso em: 28 jul. 2014.

UNITED STATES OF AMERICA. Departament of Education. National Center for Education Statistics. A Comparison of the 2011 Grade 8 NAEP and TIMSS Mathematics and Science Frameworks (NCES 2013-462). 2013. Disponível em: <http://nces.ed.gov/nationsreportcard/pubs/studies/2013462.asp>. Acesso em: 28 jul. 2014. 


\section{WALLACE NASCIMENTO PINTO JUNIOR}

Pesquisador Tecnologista em Informações e Avaliações

Educacionais da Diretoria de Avaliação da Educação Básica do Instituto Nacional de Estudos e Pesquisas Educacionais Anísio Teixeira (Inep), Brasília, Distrito Federal, Brasil

wallace.pinto@inep.gov.br 


\section{ANEXO}

QUADRO 1 - Comparativo de descritores do $9^{\circ}$ ano do Saeb e da $8^{a}$ série do Naep

\begin{tabular}{|c|c|c|c|}
\hline \multicolumn{2}{|r|}{ SAEB ( $9^{\circ}$ ano) - ESPAÇO E FORMA } & \multicolumn{2}{|r|}{ NAEP (8ª série) - GEOMETRIA } \\
\hline \multicolumn{2}{|r|}{ DIMENSÃO E FORMA } & & 8.G.1) DIMENSÃO E FORMA \\
\hline & & 8.G.1.a & $\begin{array}{l}\text { Desenhar ou descrever um caminho de menor } \\
\text { comprimento entre dois pontos para resolver } \\
\text { problemas em um contexto. }\end{array}$ \\
\hline & & 8.G.1.b & $\begin{array}{l}\text { Identificar um objeto geométrico dada uma } \\
\text { descrição escrita de suas propriedades. }\end{array}$ \\
\hline DO2 & $\begin{array}{l}\text { Identificar propriedades comuns e } \\
\text { diferenças entre figuras bidimensionais e } \\
\text { tridimensionais, relacionando-as com suas } \\
\text { planificações. }\end{array}$ & 8.G.1.C & $\begin{array}{l}\text { Identificar, definir ou descrever formas } \\
\text { geométricas no plano e no espaço tridimensional } \\
\text { dada uma representação visual. }\end{array}$ \\
\hline D06 & $\begin{array}{l}\text { Reconhecer ângulos como mudança de } \\
\text { direção ou giros, identificando ângulos retos } \\
\text { e não retos. }\end{array}$ & 4.G.1.C & $\frac{\text { Identificar ou desenhar ângulos e outras figuras }}{\text { geométricas no plano. }}$ \\
\hline \multirow[t]{4}{*}{ D11 } & $\begin{array}{l}\text { Reconhecer circulo/circunferência, seus } \\
\text { elementos e algumas de suas relaçóes. }\end{array}$ & & \\
\hline & & 8.G.1.d & $\begin{array}{l}\text { Desenhar ou esboçar polígonos, círculos ou } \\
\text { semicírculos a partir de uma descrição escrita. }\end{array}$ \\
\hline & & 8.G.1.e & $\begin{array}{l}\text { Representar ou descrever uma situação } \\
\text { tridimensional em um desenho bidimensional a } \\
\text { partir de diferentes vistas. }\end{array}$ \\
\hline & & 8.G.1.f & $\begin{array}{l}\text { Demonstrar uma compreensão sobre as formas } \\
\text { bi e tridimensionais em nosso mundo por meio de } \\
\text { identificação, desenho, modelagem, construção ou } \\
\text { decomposição. }\end{array}$ \\
\hline \multicolumn{2}{|c|}{$\begin{array}{c}\text { TRANSFORMAÇÃO DE FORMAS E PRESERVAÇÃO } \\
\text { DE PROPRIEDADES }\end{array}$} & & $\begin{array}{l}\text { 8.G.2) TRANSFORMAÇÃO DE FORMAS E } \\
\text { PRESERVAÇÃO DE PROPRIEDADES }\end{array}$ \\
\hline & & 8.G.2.a & $\begin{array}{l}\text { Identificar eixos de simetria em figuras planas } \\
\text { ou reconhecer e classificar tipos de simetrias de } \\
\text { figuras planas. }\end{array}$ \\
\hline D05 & $\begin{array}{l}\text { Reconhecer a conservação ou modificação } \\
\text { de medidas dos lados, do perímetro, da } \\
\text { área em ampliação e/ou redução de figuras } \\
\text { poligonais usando malhas quadriculadas. }\end{array}$ & \multirow{2}{*}{ 8.G.2.c } & \multirow{2}{*}{$\begin{array}{l}\text { Reconhecer ou informalmente descrever o efeito } \\
\text { de uma transformação em figuras geométricas } \\
\text { bidimensionais (reflexões a partir de eixos de } \\
\text { simetria, rotações, translações, ampliações e } \\
\text { reduções). }\end{array}$} \\
\hline \multirow[t]{4}{*}{ D07 } & $\begin{array}{l}\text { Reconhecer que as imagens de uma } \\
\text { figura construída por uma transformação } \\
\text { homotética são semelhantes, identificando } \\
\text { propriedades e/ou medidas que se } \\
\text { modificam ou não se alteram. }\end{array}$ & & \\
\hline & & 8.G.2.d & $\begin{array}{l}\text { Prever os resultados de combinar, subdividir e } \\
\text { mudar formas de figuras planas e sólidos (ex: } \\
\text { dobraduras, cobrir com azulejos (tiling), corte e } \\
\text { rearranjo de peças) }\end{array}$ \\
\hline & & 8.G.2.e & $\begin{array}{l}\text { Justificar relacões de congruência e semelhança e } \\
\text { aplicar essas relações usando escalas e raciocínio } \\
\text { proporcional. }\end{array}$ \\
\hline & & 8.G.2.f & $\begin{array}{l}\text { Para figuras semelhantes, identificar e usar } \\
\text { as relacóes de conservação dos ângulos e de } \\
\text { proporcionalidade das medidas dos lados e } \\
\text { perímetro. }\end{array}$ \\
\hline
\end{tabular}




\begin{tabular}{|c|c|c|c|}
\hline \multicolumn{2}{|r|}{ SAEB ( $9 \circ$ ano) - ESPAÇO E FORMA } & & NAEP (8a série) - GEOMETRIA \\
\hline \multicolumn{2}{|r|}{ RELAÇÕES ENTRE FIGURAS GEOMÉTRICAS } & \multirow[b]{2}{*}{ 8.G.3.b } & 8.G.3) RELAÇÕES ENTRE FIGURAS GEOMÉTRICAS \\
\hline \multirow[t]{2}{*}{ D08 } & $\begin{array}{l}\text { Resolver problema utilizando a propriedade } \\
\text { dos polígonos (soma de seus ângulos } \\
\text { internos, número de diagonais, cálculo } \\
\text { da medida de cada ângulo interno nos } \\
\text { polígonos regulares). }\end{array}$ & & $\begin{array}{l}\text { Aplicar propriedades geométricas e relações na } \\
\text { resolução de problemas simples em duas e três } \\
\text { dimensões. }\end{array}$ \\
\hline & & 8.G.3.C & $\begin{array}{l}\text { Representar situações-problema com modelos } \\
\text { geométricos simples para resolver problemas } \\
\text { matemáticos ou do mundo real. }\end{array}$ \\
\hline D10 & $\begin{array}{l}\text { Utilizar relações métricas do triângulo } \\
\text { retângulo para resolver problemas } \\
\text { significativos. }\end{array}$ & 8.G.3.d & $\begin{array}{l}\text { Usar o Teorema de Pitágoras para resolver } \\
\text { problemas. }\end{array}$ \\
\hline D03 & $\begin{array}{l}\text { Identificar propriedades de triângulos pela } \\
\text { comparação de medidas de lados e ângulos. }\end{array}$ & \multirow{2}{*}{ 8.G.3.f } & \multirow{2}{*}{$\begin{array}{l}\text { Descrever e analisar propriedades simples de, ou } \\
\text { relações entre, triângulos, quadriláteros e outras } \\
\text { figuras poligonais planas. }\end{array}$} \\
\hline D04 & $\begin{array}{l}\text { Identificar relação entre quadriláteros, por } \\
\text { meio de suas propriedades. }\end{array}$ & & \\
\hline & & 8.G.3.g & $\begin{array}{l}\text { Descrever ou analisar propriedades e relações de } \\
\text { retas paralelas ou concorrentes. }\end{array}$ \\
\hline \multicolumn{2}{|r|}{$\begin{array}{l}\text { POSIÇÃO, DIREÇÃO E GEOMETRIA DE } \\
\text { COORDENADAS }\end{array}$} & & $\begin{array}{l}\text { 8.G.4) POSIÇÃO, DIREÇÃO E GEOMETRIA DE } \\
\text { COORDENADAS }\end{array}$ \\
\hline \multirow[t]{4}{*}{ D01 } & $\begin{array}{l}\text { Identificar a localização/movimentação } \\
\text { de objeto em mapas, croquis e outras } \\
\text { representações gráficas. }\end{array}$ & & \\
\hline & & 8.G.4.a & $\begin{array}{l}\text { Descrever posições relativas de pontos e retas } \\
\text { usando as ideias geométricas de ponto médio, } \\
\text { pontos em uma reta comum que passa por um } \\
\text { ponto comum, paralelismo ou perpendicularidade. }\end{array}$ \\
\hline & & 8.G.4.b & $\begin{array}{l}\text { Descrever a interseção de duas ou mais figuras } \\
\text { geométricas no plano (ex: interseção de um círculo } \\
\text { e uma reta). }\end{array}$ \\
\hline & & 8.G.4.C & $\begin{array}{l}\text { Visualizar ou descrever a secção transversal de um } \\
\text { sólido. }\end{array}$ \\
\hline \multirow[t]{2}{*}{ D09 } & \multirow[t]{2}{*}{$\begin{array}{l}\text { Interpretar informações apresentadas por } \\
\text { meio de coordenadas cartesianas. }\end{array}$} & 4.A.2.C & $\begin{array}{l}\text { Traçar o gráfico ou interpretar pontos tendo } \\
\text { números naturais e letras como coordenadas } \\
\text { em grades ou no primeiro quadrante do plano } \\
\text { cartesiano. }\end{array}$ \\
\hline & & 8.G.4.d & $\begin{array}{l}\text { Representar figuras geométricas usando } \\
\text { coordenadas retangulares no plano. }\end{array}$ \\
\hline \multicolumn{2}{|r|}{ RACIOCÍNIO MATEMÁTICO EM GEOMETRIA } & & 8.G.5) RACIOCÍNIO MATEMÁTICO EM GEOMETRIA \\
\hline & & 8.G.5.a & $\begin{array}{l}\text { Fazer e testar uma conjectura geométrica sobre } \\
\text { polígonos regulares. }\end{array}$ \\
\hline
\end{tabular}

Fonte: Elaboração do autor com base em informações de Brasil (2002) e National Assessment Governing Board (2013). 